\title{
Parkinsonian-pyramidal syndrome
}

INSERM

\section{Source}

INSERM. (1999). Orphanet: an online rare disease and orphan drug data base.

Parkinsonian-pyramidal syndrome. ORPHA:171695

Parkinsonian-pyramidal syndrome is a rare, genetic, neurological disorder characterized by the association of both parkinsonian (i.e. bradykinesia, rigidity and/or rest tremor) and pyramidal (i.e. increased reflexes, extensor plantar reflexes, pyramidal weakness or spasticity) manifestations, which vary according to the underlying associated disease (e.g. neurodegenerative disease, inborn errors of metabolism). 